\title{
The relation between the high speed submerged cavitating jet behaviour and the cavitation erosion process
}

\author{
Ezddin Hutli ${ }^{a, b, *}$, Milos S. Nedeljkovic ${ }^{c}$, Nenad A. Radovic ${ }^{d}$, Attila Bonyár ${ }^{e}$ \\ a Institute of Nuclear Techniques, Budapest University of Technology and Economics, Budapest, Hungary \\ ${ }^{\mathrm{b}}$ Department of Thermohydraulics, Centre for Energy Research, Hungarian Academy of Sciences, Budapest, Hungary \\ ${ }^{\mathrm{c}}$ University of Belgrade, Faculty of Mechanical Engineering, Serbia \\ d University of Belgrade, Faculty of Technology and Metallurgy, Serbia \\ e Budapest University of Technology and Economics, Department of Electronics Technology, Hungary
}

\section{A R T I C L E I N F O}

Article history:

Received 21 May 2015

Revised 14 December 2015

Accepted 5 March 2016

Available online 11 March 2016

\section{Keywords:}

Cavitation

Cavitating jet

Cavitation erosion

Vortex

Shear

\begin{abstract}
A B S T R A C T
In order to accurately and reliably evaluate the cavitation erosion resistance of materials using a cavitating jet generator, the effects of the hydrodynamic parameters and the nozzle geometry on the erosion process were investigated. Since the behaviour of a high speed submerged cavitating jet is also depending on the working conditions; their influence is also discussed based on the evaluation of cavitation erosion process. The erosion rate was used as an indicator for cavitating jet behaviour. Specimens of commercialpurity copper were subjected to high speed submerged cavitating jets under different initial conditions, for certain time periods. The force generated by jet cavitation is employed to initiate the erosion in surface. The tested specimens were investigated with a digital optical microscope and a profilometer. It was found that erosion becomes more pronounced with decreasing cavitation numbers, as well as with increasing exit jet velocities. The nozzle configuration and hydrodynamic parameters have strong influences on the erosion rate, eroded area and depth of erosion. A comparison between the obtained results explains some of the mechanisms involved in cavitation and erosion processes and their relation to the tested parameters. Mathematical expressions which combine these parameters with the erosion rate are obtained. These parameters are very important in order to control the cavitation as a phenomenon and also to control the performance of the cavitating jet generator.
\end{abstract}

(c) 2016 Elsevier Ltd. All rights reserved.

\section{Introduction}

Cavitation causes many adverse effects that are to be avoided or at least controlled in any hydraulic facility. On the other hand, cavitation is used in many diverse scientific and industrial applications (jet cutting, under water cleaning, etc.) via cavitation clouds produced by cavitating jet generators. The impingement of a cavitating jet leads to serious erosion in valves and hydraulic equipment. In order to reduce cavitation erosion in valves and oil hydraulic equipment or to improve the performance of jet cutting or under water cleaning, etc., it is necessary to have an adequate knowledge about the mechanism of erosion due to the impingement of a cavitating jet (Choi et al., 2012; Field et al., 2012; Hutli et al., 2008; Soyama, 2011a; Soyama et al., 2009; Yamaguchi and Shimizu, 1987). The great advantage of testing erosion by the use

* Corresponding author at: Institute of Nuclear Techniques, Budapest University of Technology and Economics, Budapest, Hungary. Tel.: (+36-1)-463-2523; fax: (+36-1)-463-1954

E-mail address: ezddinhutli@yahoo.com (E. Hutli). of cavitating jet is that the cavitating jet apparatus can simulate different cavitating conditions. If a relationship between the cavitation intensity in a cavitating jet and the erosion rate of a material were investigated precisely, the key parameter to predict the cavitation erosion rate could be clarified and the performance of the cavitation generator could be increased as well (Hutli et al., 2010; Minguan et al., 2013; Soyama et al., 2012). The collapses of the cavities are the origin of high pressure spikes on the target surface. The amplitude of the collapse stress pulses is varying and random, but it can reach up to $1500 \mathrm{MPa}$, which is a level of stress high enough to deform or to rupture the surface of most industrial alloys (Karimi, 1986). In addition, the collapse of cavitation bubbles produces not only mechanical impact, which normally results in cavitation erosion in fluid machinery, but also high temperature spots which have a significant thermal effect.

Frequently cited studies found that a converging shock wave reflects at the bubble centre, generating peak pressures and temperatures above $10 \mathrm{Mbar}$ and $10^{7} \mathrm{~K}$ in a small central region of the bubble (Wu and Roberts, 1993; Moss et al., 1994; Bass et al., 2008). Therefore it should be noted that, in the investigation of the 
a
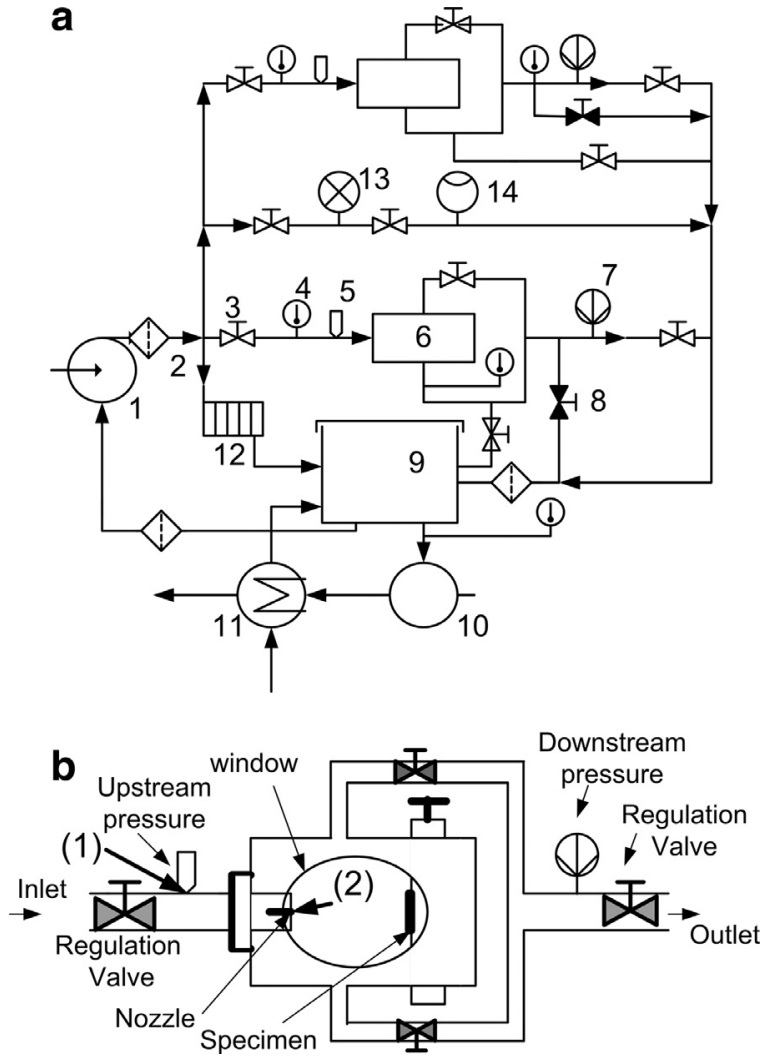

Fig. 1. (a) Schematic diagram of the cavitating jet machine. (1 - plunger pump, 2 - filter, 3 - regulation valve, 4 - temperature sensor, 5 - high-pressure transducer, 6 - test chamber, 7 - low-pressure transducer, 8 - safety valve, 9 - tank, 10 circulation pump, 11 - heat exchanger, 12 - distracter energy, 13 - pressure gauge, and 14 - flow indicator). (b) Schematic diagram of the test chamber.

cavitation damage, the possible influence of the generated temperature on the target surface should be considered.

The understanding of these mechanical and thermal effects and their application may be employed to improve the corrosion resistance of a material (Soyama and Asahara, 1999). The flow across the holes of a nozzle can be controlled by different factors, which may be classified under three categories: operation conditions, orifice geometry and flow properties. The importance of these parameters may be understood from the results of many authors who have investigated the performance of jets (e.g. Okada et al., 1995; Soyama et al., 1998a; Sun et al., 2005; Vijay et al., 1991; Zhou and Hammitt, 1983).

The aim of this study was to examine the influence of hydrodynamic conditions, such as cavitation number, exit jet velocity, and the nozzle configuration (convergent or divergent) on the behaviour of the created cavitating jet, where the cavitation erosion of commercial copper is used as an indicator of the cavitation behaviour. In this way the performance of the cavitating jet generator can be analysed.

\section{Experimental set-up and measurement procedure}

The experimental set-up for the investigation of the cavitating jet performance was the closed hydraulic loop shown in Fig. 1(a). A high speed submerged cavitating jet was produced in the test chamber by the adjustment of the appropriate hydrodynamic conditions and the final outflow to the test chamber through the nozzle. The specimens were mounted in the chamber co-axially with the nozzle, the chamber was filled with water and then the water was pressurized by a plunger pump. Only one chamber was a

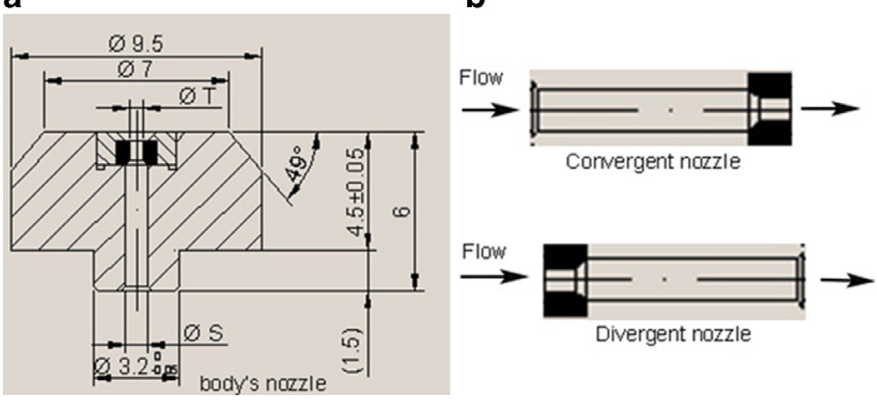

Fig. 2. (a) Geometrical parameters of the nozzle. (b) Ways of nozzle installation (dimensions in $\mathrm{mm}$ ).

functional, while the other one was in reserve. A shortcut line with a pressure gauge functioned as a pressure regulator in the system. The regulation of the water temperature to $\pm 1{ }^{\circ} \mathrm{C}$ during the erosion tests was achieved by a cooling circuit with a heat exchanger. The details of the chamber are shown in Fig. 1(b).

The hydrodynamic conditions were the following: nozzles with different diameters were used $(0.4,0.45,0.55,0.6,1,1.1$ and $2 \mathrm{~mm})$; the top speed of the jet was more than $250 \mathrm{~m} / \mathrm{s}$ for an upstream pressure set to 450 bar (with nozzle diameter of $0.45 \mathrm{~mm}$ ); the downstream pressure could be adjusted from atmospheric pressure up to 6 bar. The volume of the test chamber was 0.871

A rotating holder was employed to attach up to 6 specimens at a distance of $x=25.67 \mathrm{~mm}$ away from the nozzle (on the opposite side of the chamber - Fig. 1(b)), with the aim to allow switching from one sample to another during the test. It also allows a quick start and/or stop of the exposure to cavitation without the necessity for turning on/off the test rig. The software used for data acquisition and to control the machine was LABVIEW 7.1.

Usually the non-dimensional standoff distance $\left(\frac{x}{d}\right)$ where $d$ is the outlet nozzle diameter is used instead of standoff distance $(x(\mathrm{~mm}))$.

Before the tests, the specimens were prepared by metallographic polishing in order to provide a perfectly smooth surface appropriate to be examined for any damage imposed by the cavitation jet.

The specimens were first weighed and then mounted in the holder. The hydrodynamic conditions were selected to produce a suitable cavitating jet. The cavitating jet then impinged on the specimen at $90^{\circ}$ to its surface. The facility was turned off, the chamber evacuated and then the specimen was removed. The specimens were dried and weighed and then the procedure was repeated with other specimens. The intensity of the cavitating jet was controlled via the upstream and downstream pressures, which were measured precisely by transducers and controlled using the needle valves (regulation valves). Filters were employed to remove impurities from the circulating water. A temperature regulator and temperature sensors were used to control the water temperature. Fig. 2 shows the geometrical properties of the nozzle mounting. The nozzle could be mounted in the holder in two ways with respect to the inlet and outlet diameters: divergent and/or convergent conical nozzle.

\section{Cavitation erosion parameters and erosion quantification}

The cavitation number $\sigma$ was calculated as $\sigma=$ $\left(p_{\text {ref. }}-p_{\mathrm{v}}\right) /\left(0.5 \rho V_{\text {ref. }}^{2}\right)$. It represents a measure of resistance of the flow to the incidence of cavitation, since the lower it is, the more intensive the cavitation will be. The value of this parameter was obtained by measuring the upstream and downstream pressures, and by calculating the reference velocity from the measured flow-rate. $p_{\text {ref }}$ is the reference pressure $\left(p_{2}\right), p_{\mathrm{v}}$ is the 
vapour pressure, $\rho$ is the density, and $V_{\text {ref }}$ is the reference velocity $\left(V_{\text {ref }}=\right.$ exit jet velocity $\left.\left(V_{\mathrm{J}}\right)\right)$. At regular intervals, the specimen was carefully removed from the chamber, dried sufficiently and then submitted to further analysis. Usually, the rate of mass loss, the rate of volume loss or the rate of the mean depth of penetration are used as good measures of the erosion intensity. In this paper, the erosion rate $E . R .=\Delta W / \Delta t$ was used as the index to quantify the erosion, where $W$ is the mass of the specimen (Soyama et al., 1998b). In addition, a profilometer was used to quantify the erosion area (cutted) and the depth of the cutting.

\section{Error analysis of the measured quantities (experimental uncertainty)}

All the apparatus in the facility (cavitating jet generator) were calibrated in order to obtain results with a high accuracy. The pressure transducers used to measure the upstream and downstream pressure were calibrated precisely with a reference pressure transducer (HUBER), then the flow rate for the different nozzle diameters and different directions of flow were measured precisely in order to obtain the constant $k$ in different cases for the calculation of the exit jet velocity $\left(V_{\mathrm{J}}\right)$. The temperature sensors in the test rig were calibrated perfectly by the use of a NORMA type a digital thermometer as the reference in the calibration process. The upstream pressure $\left(p_{1}\right)$ and the downstream pressure $\left(p_{2}\right)$ were measured at the inlet and outlet of the test chamber, respectively. The pressure transducers were calibrated by the manufacturer and accuracy certificates were issued for a maximum error of $+0.2 /-0.21$ $\% \mathrm{FS}$. As the flow rate was determined by using the $P_{1}$ and $P_{2}$ values from a previous nozzle calibration, the uncertainty in the determination was also of the order of $\pm 0.3 \% \mathrm{FS}$. In order to assess the reproducibility and error reduction of the erosion loss, three specimens were exposed to the cavitation in each step. The average value of loss was used for further calculations. The mass loss was measured by using a high precision balance (METTLER AE 100), with a sensitivity of $0.1 \mathrm{mg}$.

\section{Basic mechanism of the cavitation phenomenon generated by a cavitating jet generator}

When the jet penetrates the stagnant water in the test chamber, a shearing force is generated between them (the shearing force is depending on the velocity gradient), which results in the generation of a vortex. The existence of a vortex can be verified and tested by many parameters such as the circulation parameter $(\Gamma)$, the vorticity $(\omega)$, the centrifugal force $\left(F_{\mathrm{c}}\right)$ and the energy of the vortex. The change from a constant vorticity to a radially varying vorticity is much sharper for a turbulent vortex ring. In a turbulent field, the presence of additional vortices with random circulations in the neighbourhood will disturb the closed streamline pattern around any vortex (Soyama et al., 1998b). However, in general, in a turbulent flow (single phase), the portions of the fluid move both horizontally and vertically and there is a continuous "mixing of particles". Some move upwards and some downwards according to a stochastic process. Portions of fluid move irregularly causing a continuous exchange of momentum from one portion of the fluid to another and this momentum exchange is the reason for the turbulent shear stress, which is also called the Reynolds stress. In the case of cavitating jets (two phase flow), part of the momentum exchange is established by the change of the phase (density change), and also, because the Prandtl mixing length theory, which is based on the assumption that a fluid parcel has to travel over a length $l_{\mathrm{m}}$ before its momentum is transferred. Basically, for single phase flow it is easy to understand the concept of the mixing length: since turbulent stress is caused by momentum transfer, it is useful to have a physical length that defines when the momentum has been transferred (Agrawal and Prasad, 2003).
For two phases, highly turbulent flow, as in the cavitating jets, this is more complicated. Maybe, it can be assumed that the mixing length is equal to the width of the jet at a given point, which can be used as an indicator for jet spreading. In the case of cavitating jets created by a circular nozzle, the initial vortex of the jet produces a nearly axisymmetric low pressure. This low pressure leads to the formation of ring-like cavitation bubbles in the near field of the jet. In the early stages of jet development process, cavitation bubbles, which are generated inside the starting vortices, are sometimes connected with each other in the form of a ring. The bubble rings travel downstream with the initial vortices and often survive for a certain distance (Hutli et al., 2013). In the later stages of jet development, isolated bubbles are formed behind the initial vortices; some of them are formed in groups, while others are randomly distributed in the shear layer of the jet. The stability of a vortex cavitation ring is depending on the intensity of turbulence (injection pressure and velocity distribution), the pressure in the test chamber and the stage life of the bubbles (rebounding and collapsing). As the exit velocity is increasing (assuming the other parameters are constant), the vortex generation rate increases, which means an increased number of cavitation bubbles. Also, as the downstream pressure (pressure inside the test chamber) decreases, the rate of vortex generation increases because the jet can easily rotate the stagnant liquid and produce a vortex (Hutli et al., 2013). In the case of high speed, continuously submerged cavitating jets, it is a complicated task to describe the mechanism of vortex creation, since there is a two phase flow in addition to the rebounding and collapsing of the cavitation bubbles, which frequently occurs along the jet trajectory producing very fast microand nano-jets and shock waves. These liquid jets produce many new vortexes; in fact, thereby turbulent flow and chain vortex production exist (Hutli et al., 2013; Katepalli, 1999). Fig. 3 shows examples for the vortex rings in several different cases: in the simple case of an air jet (pulsed jet) (a), a submerged liquid jet in the same type of liquid (no cavitation) (c), a submerged cavitating jet (pulsed) (Dynaflow, Inc., 2016.) (c) and a submerged cavitating jet (continuous) (d). In the air jet it can clearly be seen how the vortex rings shift positions during their movement.

\section{Flow characteristics of a cavitating jet (cavitation clouds)}

The flow structure in the near field of the jet and in the jet itself is complex. As it is well known, the cavitating flow is treated as a two-phase flow, which consists of a continuous liquid phase and a dispersed gaseous phase in the form of bubbles in cavity clouds. In this kind of flow a relative motion exists between the bubbles and the surrounding fluid. In many cases, the transfer of mass and/or heat is important. Cavity flows behave very differently from single-phase flows. The presence of a second phase with significantly different density, viscosity and elasticity considerably alters the effective properties of such a mixture. The interfaces between the liquid and the gas phase are deformable and unsteady. Encounters between bubbles can lead to bouncing or coalescence. Bubbles may accelerate, deform, execute shape or volume oscillations or even break up (collapse) (Sridhar and Katz, 1995). Since in our case a continuous jet is used, the cavitation can be treated as a fast phenomenon (varying in order of few $\mu \mathrm{s}$ ), the vortex ring does not appear clearly, and also the oscillation and collapse of the individual bubbles cannot be seen. In order to describe the flow characteristics, because of the reasons mentioned earlier, we can follow only the shedding and discharging of the cavity clouds, in a macro-level. As it is already shown in pervious publications, the characteristic unsteady motion of cavitation clouds can be perceived through the observation of the cavitating area by using a high-speed video camera $(100,000 \mathrm{f} / \mathrm{s})$. It was noted that the shedding pattern has a periodic character, although it does not stay 

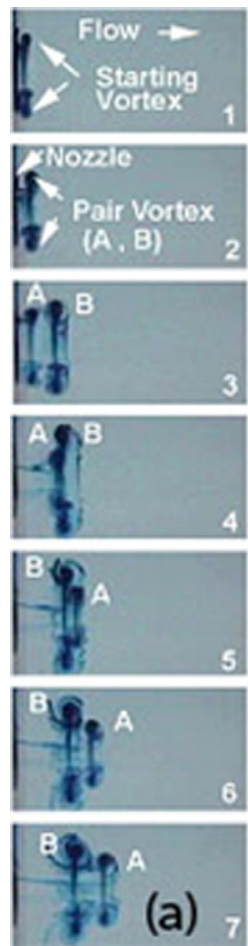
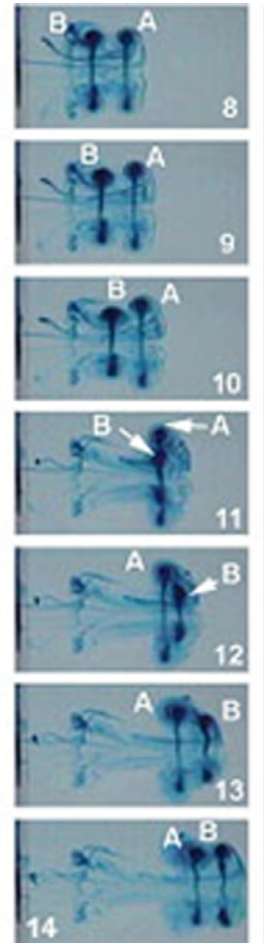

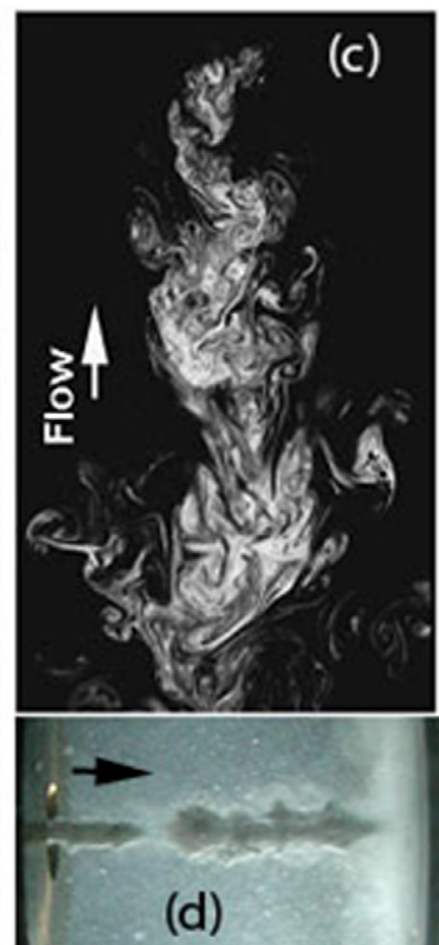

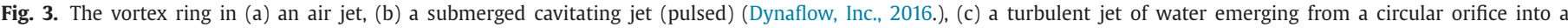
tank of still water (one phase) (Katepalli, 1999), and (d) a submerged cavitating jet (Hutli et al., 2013).

a

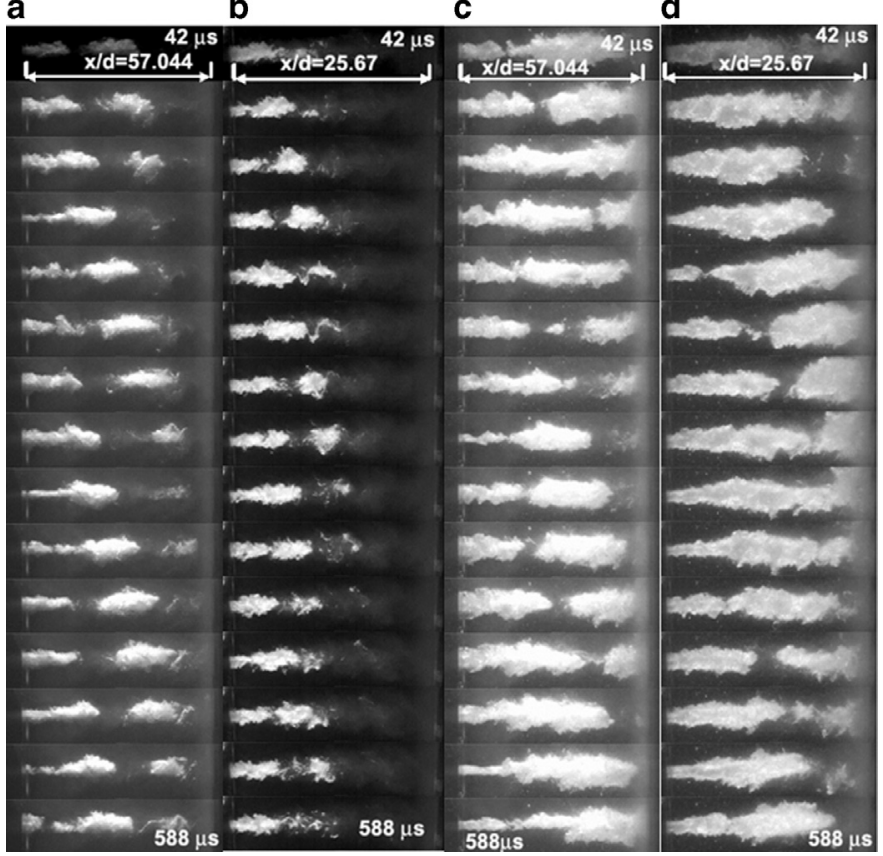

Fig. 4. High-speed camera images $(24,000 \mathrm{f} / \mathrm{s}, 512 * 128)$ of the shedding patterns. (a) Convergent nozzle $\left(P_{1}=105 \mathrm{bar}, P_{2}=2.06 \mathrm{bar}, V_{\mathrm{J}}=96.5 \mathrm{~m} / \mathrm{s}, \sigma=0.044\right.$, $\left.T=18.5^{\circ} \mathrm{C}\right)$. (b) Divergent nozzle $\left(P_{1}=90.5\right.$ bar $P_{2}=1.89 \mathrm{bar}, V_{\mathrm{J}}=23.4 \mathrm{~m} / \mathrm{s}, \sigma=0.69$, $\left.T=20^{\circ} \mathrm{C}\right)$. (C) Convergent nozzle $\left(P_{1}=177 \mathrm{bar}, \quad P_{2}=2.06 \mathrm{bar}, \quad V_{J}=125.7 \mathrm{~m} / \mathrm{s}\right.$, $\left.\sigma=0.026, T=18.5^{\circ} \mathrm{C}\right)$. (d) Divergent nozzle $\left(P_{1}=267 \mathrm{bar}, P_{2}=1.89 \mathrm{bar}, V_{\mathrm{J}}=40.5 \mathrm{~m} / \mathrm{s}\right.$, $\sigma=0.23, T=20^{\circ} \mathrm{C}$ ). The flow direction is from left to right (Hutli and Nedeljkovic, 2008).

regular for a long time and it may change with the variation of the working conditions as can be seen in Fig. 4. The imperfect shedding pattern could be the result of the incompatibility between the test equipment (nozzles, feed pumps, test chamber, and recording system) (Hutli and Nedeljkovic, 2008). In general as it appears, the cloud expands almost to the middle of the trajectory, which distance depends on the working conditions. The leading part of the main cavitating jet moves gradually toward the target and then the jet shows a shrinking motion in the diameter close to the exit of the nozzle, as can be clearly seen in the case of convergent nozzle in Fig. 4.

This shrinking motion in the cloud diameter does not appear clearly in the case of a divergent nozzle, while for a convergent nozzle this phenomenon usually could be observed. This cloud shrinking motion moves toward the upstream direction. At the same time some downstream clouds, which have already shrunk, change to a growing stage and translate toward the downstream direction. This re-entrant motion reaches the nozzle exit and then changes to a new shedding motion of a cavitation cloud in the reverse direction. As it appears in some images, there are discontinuous parts of the cavitation clouds, which are caused by the arrival of the reentrant motion at the nozzle exit (Fig. 4). The leading part a of new cavitating cloud, defined at this point of discontinuity, moves downstream with a certain speed depending on the working conditions. The analysis of long recordings (some hundreds frames obtained with a shutter time of $20 \mu \mathrm{s}$ ) revealed that the cavitation phenomenon appears to have a chain reaction behaviour. As a result, the new cavitating jet grows and develops in a coalescing manner with some already existing clouds and then moves downstream from the exit of the nozzle. Also it could be noticed from Fig. 4, that the cavity clouds do not keep their shape, they are changing with time and position, which changes are related to the forces acting on the clouds. In general, the forces acting on the bubble or on the cavity cloud are composed of separate and uncoupled contributions from pressure gradient, drag, lift due to vorticity, virtual mass, hydrodynamic interaction forces (hydrodynamic interactions between adjacent bubbles) and gravity (Sridhar and Katz, 1995). Therefore, as the clouds are subjected to static and dynamic forces, their volume and area are changing, thus the calculated speed $(V)$ does not exactly represent the jet speed $\left(V_{\mathrm{J}}\right)$. 
a

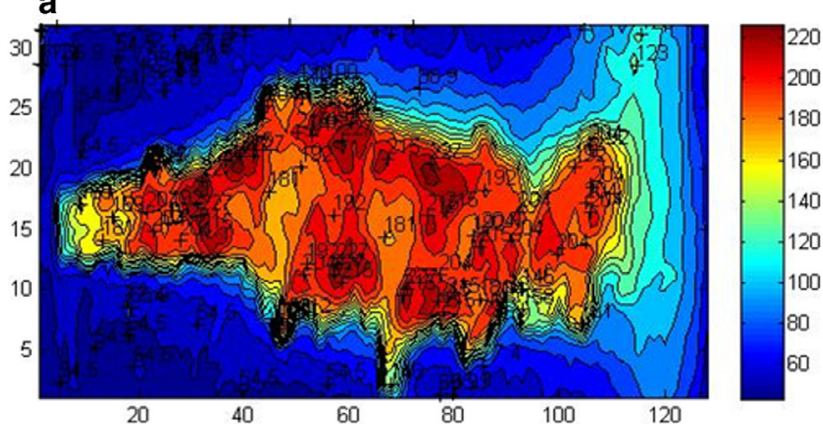

b

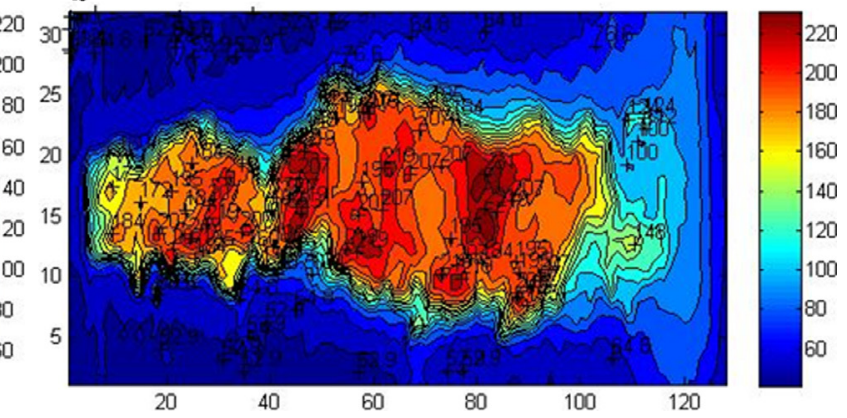

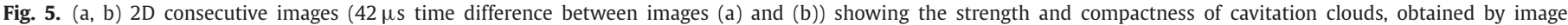
processing. (Divergent nozzle $\left(D_{\text {in }}=0.45\right.$ and $\left.D_{\text {out }}=1 \mathrm{~mm}\right)\left(P_{1}=267 \mathrm{bar}, P_{2}=1.89 \mathrm{bar}, V_{\mathrm{J}}=40.5 \mathrm{~m} / \mathrm{s}, \sigma=0.23, T=20^{\circ} \mathrm{C}\right.$.)

The effects of the geometrical and hydrodynamic parameters can be understood based on Fig. 4. In the case of a divergent nozzle with low injection pressure (Fig. 4(b)) the shedding of cloudlike cavity may be depending on the weakening of vortex formation and the increase of three dimensional disturbance on the separated shear layer (Sato and Yasuhiro, 2003). But in the case of high injection pressure for both cases (convergent and divergent), the reason for the unclear distinguishing between both shedding and discharging processes is related to a strong vortex formation, the inherent compact behaviour of the shear layer and the length of cavitating area, and in addition to the fast formation of vortex cavitation and their interaction with each other. Based on this result it should be noted that the shedding frequency at low pressures and the influence of nozzle geometry remains to be further examined from the point of view of vortex behaviour on the shear layer.

Cloud dynamics, which gives information about the strength and compactness of objects (cavitation clouds) is obtained through image processing at pixel level using a special program with Matlab. As can be seen in Fig. 5 the changes in the intensity reflect the changes in the size and shape of the cavitation bubbles. However, due to the scattering of the light at the gas-liquid interface the edges of the bubbles can be blurred and hard to precisely indentify.

Also the translational speed of the leading part of the cavitating jet was calculated by using a given number of consecutive frames (with a known frame rate) and by measuring the differences between the positions of the leading part of cavity clouds in the consecutive frames. It was found that in the beginning the speed remains constant, then acceleration and de-acceleration processes take place. As a result a new pressure distribution map is assumed to be formed. In fact this speed does not represent the real speed of the cavity clouds, since the cavitating jet is varying during its life, i.e. it undergoes in many process as mentioned earlier.

\section{Fluid-solid interaction and cavitation damage model}

The scenario of solid and fluid interaction in the case of cavitating jets could be explained in two levels: macro- and micro-scale levels. In the macro-scale level the clouds and rings are considered as one unit, which, at the beginning of the test, hits the surface of the specimen. The contact surface, where the cavitation cloud hits the sample has a ring shape. In this area of contact the force of the cavitation cloud can be resolved into two components, normal and shear. The shear component in the specimen can either move it or, in the case of a mounted sample can produce plastic deformation. Therefore, the plastic deformation is localized in the area of the ring. With time the deformation becomes more pronounced.

In the micro-level scenario as our model illustrates in Fig. 6 (based on the assumption that the cloud is composed of small bubbles), the cavitation cloud ring collapses at the moment of impact

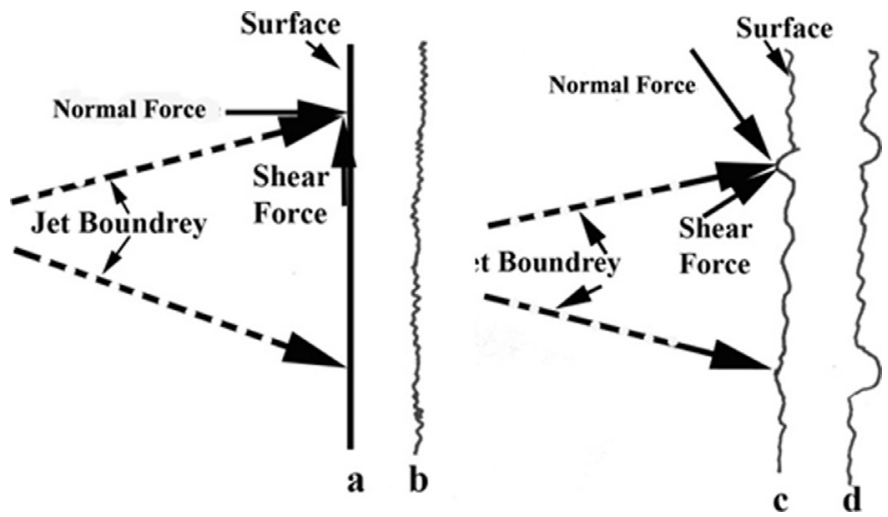

Fig. 6. Sketch of the specimen surface during erosion test: (a) start of the test: contact between cavitating jet and smooth specimen; (b) roughened surface after deformation on the surface; (c) contact between cavitation cloud and roughened specimen; and (d) start of rupturing on specimen surface.

with the surface of a specimen. At that very moment, the cavitation ring becomes divided into a large number of small bubbles. The newly formed bubbles and which resemble the cavitation cloud are neither equal in size or in strength. The space between them is very small, allowing the liquid to flow between them and to accelerate (micro-jets). This acceleration leads to an increase in kinetic energy. Due to differences in the sizes and positions of the bubbles, when they collapse, the formed "micro-jets" now hit the specimen at angles other than $90^{\circ}$, thus introducing a shear stress component at the surface, as can be seen in Fig. 6(a). This shear component seems to be sufficient to initiate plastic deformation on the surface of the specimen. After this initial step, further microjets hit the roughened surface, Fig. 6(b) and (c), leading to the commencement of rupturing of the surface and finally to its erosion (Fig. 6(d)).

It is not easy to predict the number of the bubbles in the cavitation cloud, especially when the cavitating jet is continuous, therefore we should deal with the cloud as one unit. The strength of the clouds is the function of many parameters such as bubble content, shape of the bubbles, number of bubbles in the cloud, pressure around the cloud, working fluid temperature, forces acting on the cloud, etc. The micro-jets and shockwaves are results of bubble collapsing. The velocity of the micro/nano-jets is a function of the position of the bubble collapse. Some literature reported that the maximum jet velocities are between 50 and $100 \mathrm{~m} / \mathrm{s}$. Reported values of the local material stress induced by the micro-jets are from 100 to over $1000 \mathrm{MPa}$ (Karimi and Martin, 1986). Others a maximum jet velocity of $950 \mathrm{~m} / \mathrm{s}$ (Emil et al., 2001).

Fig. 7 shows the deformation stages caused by cavitation on the target surface. By increasing the exposure time, the damage done 

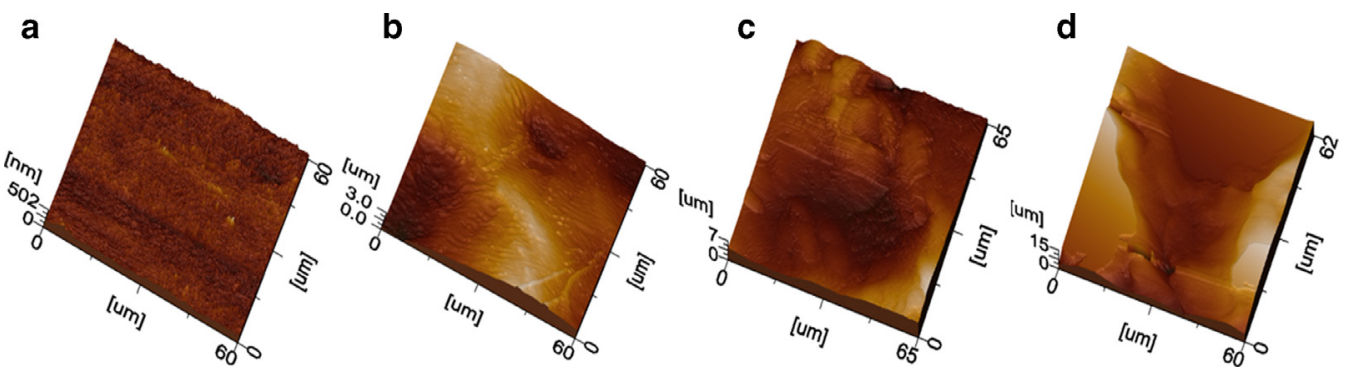

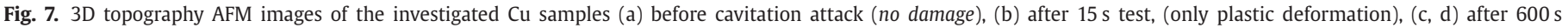
test ((d) cavitation erosion). The images are not from the same point on the surface (Hutli et al., 2013).

Table 1

Working conditions - influence of the cavitation number $(\sigma)$.

\begin{tabular}{|c|c|c|c|c|c|c|c|}
\hline Parameter & \multicolumn{7}{|c|}{$\begin{array}{l}\text { Convergent nozzle }\left(T=21^{\circ} \mathrm{C}, X / d=57.044 \text {, }\right. \\
\text { exposure time }=1 \mathrm{~h})\end{array}$} \\
\hline$P_{2}[$ bar $]$ & 2.87 & 2.78 & 2.67 & 2.6 & 2.49 & \multicolumn{2}{|l|}{2.25} \\
\hline$P_{1}$ [bar] & 175.5 & 175.7 & 175.5 & 175.5 & 175.5 & \multicolumn{2}{|l|}{175.5} \\
\hline$\sigma$ [dimensionless] & 0.037 & 0.035 & 0.034 & 0.033 & 0.032 & \multicolumn{2}{|l|}{0.029} \\
\hline$V_{\mathrm{J}}[\mathrm{m} / \mathrm{s}]$ & \multicolumn{7}{|c|}{125.05} \\
\hline Parameter & \multicolumn{7}{|c|}{ Divergent nozzle $\left(T=21^{\circ} \mathrm{C}, X / d=25.67\right.$, exposure time $\left.=1 \mathrm{~h}\right)$} \\
\hline$P_{2}$ [bar] & 2.41 & 2.55 & 2.65 & 2.71 & 2.92 & 3.2 & 3.83 \\
\hline$P_{1}$ [bar] & 246.9 & 247 & 247.2 & 247.6 & 247.9 & 248 & 248.3 \\
\hline$\sigma$ [dimensionless] & 0.32 & 0.34 & 0.35 & 0.36 & 0.39 & 0.42 & 0.51 \\
\hline$V_{\mathrm{J}}[\mathrm{m} / \mathrm{s}]$ & & & & 38.9 & & & \\
\hline
\end{tabular}

by cavitation can move beyond plastic deformation. Erosion may occur which removes material from the sample surface as observed in this case of $\mathrm{Cu}$ sample after $600 \mathrm{~s}$ exposure time. The determination of incubation time (which is needed to reach the erosion stage) or studying the effect of the exposure time on the cavitation damage (surface topology and cavitation erosion) is beyond the scope of our current paper. In order to show the earlier stages of cavitation damage Fig. 7 shows the nano- and micro-level of the cavitation damage and the characteristic features on the specimen's surface with Atomic Force Microscopy (AFM) images. These measurements were done on a JEOL SPM 5200 in room temperature at the NanoLab, University of Belgrade, Faculty of Mechanical Engineering. More information regarding these experiments can be found in our earlier publication (Hutli et al., 2013).

\section{Results and discussion}

\section{Influence of the cavitation number $(\sigma)$}

In order to investigate the influence of the cavitation number $(\sigma)$ and/or the down-stream pressure $\left(p_{2}\right)$ on the jet characteristics and on the cavitation erosion process, commercial $\mathrm{Cu}$ specimens were exposed to the cavitating jet at different cavitation numbers (different degrees of cavitation i.e. different cavitation intensities). The exposure time was one hour for each specimen. The cavitation number $(\sigma)$ was controlled by changing of downstream pressure $\left(p_{2}\right)$, via the regulation valves mounted in the outlet pipes at the test chamber (as can be seen in Fig. 1(b)). Table 1 collects the applied working conditions. Selection of the values of the cavitation number $(\sigma)$ was guided by experience gained in the use of a cavitating water jet generator for the production of cavitation erosion. The other parameters, such as the upstream pressure $\left(p_{1}\right)$, jet-velocity $\left(V_{\mathrm{J}}\right)$, fluid temperature $(T)$, nozzle outlet diameter $\left(d_{\text {out }}\right)$, nozzle geometry (convergent or divergent), non-dimensional standoff-distance $(x / d)$ and non-dimensional aspect ratio $\left(L / d_{\text {out }}\right)$, were kept constant for each nozzle during this investigation. In order to reduce the error, three specimens were used for each cavitation number and the average of the three erosion rates was used.
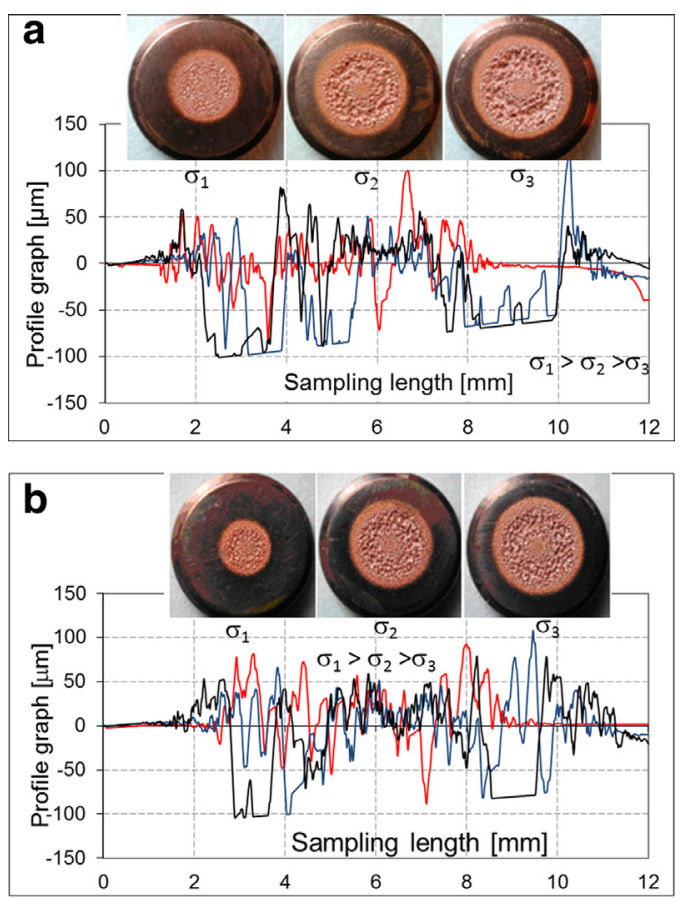

Fig. 8. Optical microscopy images and cross-sectional surface profiles illustrating the influence of the cavitation number on the erosion: (a) convergent nozzle ( $\sigma=0.037,0.034$, and $0.029\left(\sigma_{1}, \sigma_{2}, \sigma_{3}\right.$, respectively), and (b) divergent nozzle ( $\sigma=0.51,0.36$ and 0.32 , respectively) (see Table 1 ). Note that the bottom straight lines in the graph indicate that the roughness is out of the measurement range.

The eroded specimens after different treatments in this experiment are shown in Fig. 8(a) and (b). It is clear that with decreasing cavitation number (the exit jet velocities were constant at $125 \mathrm{~m} / \mathrm{s}$, $38.9 \mathrm{~m} / \mathrm{s}$ for convergent and divergent nozzles, respectively) the damaged area increased, both in total surface area and in depth.

For higher cavitation numbers in the case of convergent nozzle (Fig. 8(a)), the erosion shifted towards the centre instead of outwards. This is attributed to the decrease of the jet width (jet spreading angle) with increasing $\sigma$ (due to the increase in $P_{2}$ ), i.e. when the cavitation number increases, the jet becomes narrower. Therefore, the shedding vortices (shedding cavities) decrease and the cavity clouds (cluster of bubbles produced) collapse before reaching the specimen surface (jet penetration decreases as $\sigma$ increases). The production of cavitation is increased by decreasing $P_{2}$, since the pressure inside the vortices will arrive at $p_{\mathrm{v}}$ more easily, according to the expression $P_{\min }=P_{\mathrm{a}}-\rho \Gamma / 4 \pi^{2} r_{\mathrm{c}}^{2}$, where $\Gamma$ is the circulation parameter of the viscous core of radius $r_{\mathrm{c}}, P_{\mathrm{a}}=P_{2}$ and $P_{\text {min }}$ represents the minimum pressure in the axis of the vortex (Kalumuck, 1994).

A similar behaviour can be noticed in Fig. 8(b), i.e. in specimens tested with the divergent nozzle. In the case of the 

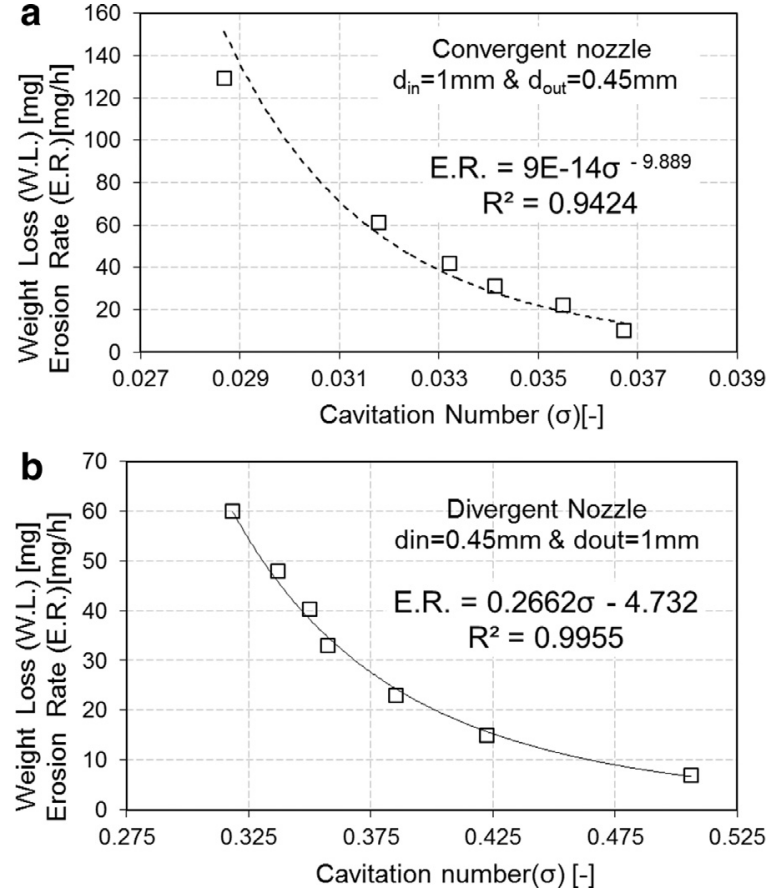

Fig. 9. Influence of the cavitation number on the erosion rate of pure $\mathrm{Cu}$ : (a) convergent nozzle and (b) divergent nozzle.

divergent nozzle, the nozzle was mounted in the correct direction $\left(d_{\text {in }}=0.45 \mathrm{~mm}, d_{\text {out }}=1 \mathrm{~mm}\right)$, while in the case of the convergent nozzle when it was mounted in the opposite direction $\left(d_{\text {in }}=1 \mathrm{~mm}\right.$, $d_{\text {out }}=0.45 \mathrm{~mm}$ ). The correct direction (divergent conic) of mounting the nozzle is the way which was proposed by the manufacturer for the use of water (liquid) jet cutting. In general, the results revealed that the damage had the same circular/ringed pattern as in the case of the convergent nozzle. On the other hand, the damage was less pronounced with the divergent nozzle than with the convergent one, as indicated by the lower surface roughness. The reason for this difference can be attributed to the large difference in the exit-jet velocity (i.e. to the large difference in the impact rate).

Fig. 8(a) and (b) shows also the cross-sectional profile graphs (as quantification of the erosion area and depth of the erosion) for different velocities and nozzle geometries (convergent or divergent). The specimens exposed to the cavitating jet produced by the convergent nozzle were rougher than those exposed to the cavitating jet produced by the divergent nozzle. The erosion rings can be easily recognized based on the optical microscopy images. These rings show different degrees of damage which can be related to many reasons, such as bubble distribution and strength and the pressure distribution that let the bubbles to collapse.

In order to quantify erosion, the erosion rate was calculated. The influence of the cavitation number is shown in Fig. 9(a) and (b) for both the convergent and divergent nozzles. As we have mentioned earlier we are using the classical definition for calculating the cavitation number $(\sigma)$; therefore we have to keep both the velocity and downstream pressure constant in order to obtain the same cavitation number under the assumption that the other parameters in the definition are constant. The standoff distance between the target and the nozzle lip was fixed. The comparison between nozzles will not be discussed here in order to avoid the confusion. In this work we would like to investigate the influence of the hydrodynamic parameters on the cavitation erosion, rather than the effect of the geometrical parameters, although we used different nozzles in the experiments. In all of the cases, the erosion rate decreased with increasing cavitation number, for both the convergent and divergent nozzles. The trend was more pronounced in the case of the convergent nozzle: it is clear that the weight loss in the case of the convergent nozzle was almost double compared to the divergent one. In addition, the influence of nozzle diameter could be noticed in Fig. 9(a) and (b). The differences in the measured erosion rates between the two nozzle types can be attributed to the parameters which are affected by the differences in the nozzle geometries. These parameters are: exit jet velocity, fluid flow geometry and pressure distribution in the test chamber (Choi et al., 2012; Soyama et al., 2009; Hutli et al., 2010; Soyama et al., 2012; Minguan et al., 2013; Soyama, 2011a; Benajes et al., 2004; Nurick, 1976). These parameters also have a significant influence on the starting position of the cavitation phenomenon (inside or outside of the nozzle), the cavitation intensity, the jet behaviour, the impact rate and the distribution of the cavity bubbles along the jet coordinates $(r, x)$ of the jet trajectory, as can be seen in Fig. 4 (Hutli and Nedeljkovic, 2008). In the case of the divergent nozzle, the cavitation is assumed to commence inside the nozzle. This is attributed to the fact that the geometry of the nozzle has a significant influence on the flow pattern inside the nozzle (Minguan et al., 2013; Hutli and Nedeljkovic, 2008; Vijay et al., 1991; Nurick, 1976). Fig. 9(a) and (b) shows that, the relation between the erosion rate and the cavitation number $(\sigma)$ is a power type relation as it can be seen on the labels in Fig. 9(a) and (b).

\section{Influence of the exit-jet velocity}

In order to understand the influence of the exit jet-velocity on the cavitation erosion process, pure $\mathrm{Cu}$ specimens were exposed to the cavitating jet at different exit jet-velocities. The investigations were performed with convergent and divergent nozzles and with different diameters $\left(d_{\text {in }}\right.$ and $\left.d_{\text {out }}\right)$. The cavitation number $(\sigma)$ and fluid temperature $(T)$ were maintained constant in every case. (The given exit velocities $\left(V_{\mathrm{j}}\right)$ are calculated based on a single phase flow. For the same mass flow rate, the actual flow velocity will be higher.) The downstream pressure was changed in order to keep the cavitation number constant. The exposure time was one hour for each specimen, to ensure measurable amounts of erosion with these working conditions. The exit jet-velocity was controlled by changing the upstream pressure $\left(P_{1}\right)$ via regulation valves, which were mounted in the inlet pipe of the test chamber and in other parts of the test rig, as shown in Fig. 1. The values of the employed up- and downstream pressures are given in Table 2. Three specimens were used for each testing point (each velocity) and the average of the erosion rate was used.

Eroded specimens after different treatments and the related cross-sectional profiles are shown in Fig. 10(a) and (b) for convergent and divergent nozzles, respectively. It is clear that with increased exit jet velocity, the damaged area increased. This increase was more pronounced in depth than in total surface area. This behaviour is related to the frequency of impact, which increases as the jet velocity increases (Soyama, 2011a; Soyama et al., 2009, 2012; Hutli et al., 2010; Hutli and Nedeljkovic, 2008; Minguan et al., 2013). Also, producing cavitation can be easily achieved by increasing the jet velocity, since the pressure inside the vortices decreases and reaches $p_{\mathrm{v}}$ at the working temperature more easily, according to the expression $P_{\min }=P_{\mathrm{a}}-\rho \Gamma / 4 \pi^{2} r_{\mathrm{c}}^{2}$ (Kalumuck, 1994). Here, $\Gamma$ increases with increasing jet-velocity (shear increases) and thus the production rate of cavities (bubbles) is increased since the shear zone will be thicker and faster in the production process. The specimens which were attacked by using the divergent nozzle showed much smaller damage on the surface than those attacked with the convergent nozzle, presumably due to the lower jet velocities. Again, increasing the exit jet velocity leads to an increase in both the eroded surface area and roughness. The erosion rate as function of exit jet velocity is shown in Fig. 11(a) and (b) for both 
Table 2

Hydrodynamic working conditions - the influence of the exit-jet velocity.

\begin{tabular}{|c|c|c|c|c|c|c|c|c|c|c|c|}
\hline \multirow{2}{*}{$\begin{array}{l}\text { Parameter } \\
P_{1}[\mathrm{bar}]\end{array}$} & \multicolumn{5}{|c|}{ Convergent nozzle $\left(T=21^{\circ} \mathrm{C}, X / d=57.044\right.$, exposure time $\left.=1 \mathrm{~h}\right)$} & \multicolumn{6}{|c|}{ Divergent nozzle $\left(T=21^{\circ} \mathrm{C}, X / d=25.67\right.$, exposure time $\left.=1 \mathrm{~h}\right)$} \\
\hline & 166 & 182 & 199 & 210 & 230 & 153 & 183 & 204 & 253 & 261 & 303 \\
\hline$P_{2}$ [bar] & 3.1 & 3.4 & 3.71 & 4.05 & 4.28 & 2.9 & 3.55 & 4.25 & 4.72 & 5.2 & 5.7 \\
\hline$V_{\mathrm{J}}[\mathrm{m} / \mathrm{s}]$ & 121.3 & 127 & 132.9 & 136.4 & 142.8 & 30.5 & 33.3 & 35.1 & 39.2 & 39.8 & 42.9 \\
\hline$\sigma$ [dimensionless] & & & & 0.042 & & & & & 64 & & \\
\hline
\end{tabular}
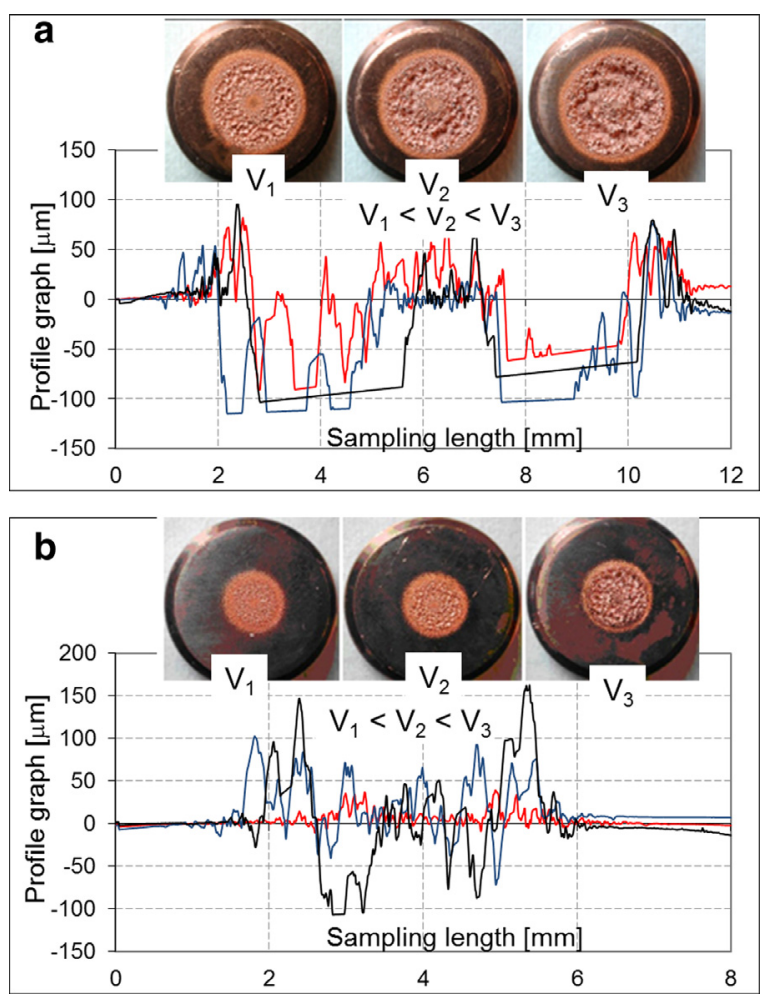

Fig. 10. Optical microscopy images and cross-sectional surface profiles illustrating the influence of the exit jet velocity on the erosion process in pure $\mathrm{Cu}$; (a) convergent nozzle: $V_{\mathrm{J}}=121.3,132.9$, and $142.8 \mathrm{~m} / \mathrm{s}\left(V_{1}, V_{2}, V_{3}\right.$, respectively), and (b) divergent nozzle: $V_{J}=30.5,35.1$ and $42.9 \mathrm{~m} / \mathrm{s}$. (see Table 2). Note that the bottom straight lines in the graph indicate that the roughness is out of the measurement range.

nozzle types. The depth of the pits increased with higher velocities due to the higher frequency of impacts and the higher kinetic energy of micro-jets which are produced during bubble collapsing on the specimen surface. In addition, the eroded area increased with increasing jet velocities, which was most probably due to the increasing jet-width (spreading angle and the shear zone). Further evaluation of the damaged surfaces revealed that the cavitation intensities varied strongly along the diameter of the cavitating jet. Therefore, it may be assumed that there is a possibility to predict the location of the bubble implosion as a function of the jet velocity, cavitation number, and to predict the distribution of bubbles with respect to their strength (implosion pressure) along the jet path and jet radial cross-section. This conclusion could help in the optimization of cavitating jet performance for industrial applications such as pinning, cutting, etc. The erosion rate is strongly depending on the exit jet velocity (Figs. 10 and 11). It can be concluded that the width of the cavitating jet increases with the velocity together with the frequency of the shedding vortices; thus the cavities and bubbles increase in both number and strength (Choi et al., 2012; Hutli et al., 2010, 2013; Minguan et al., 2013; Fujikawa, 1998; Soyama, 2005, 2011b; Soyama et al., 1994, 1995, 2009, 2012; Kato and Shimomura, 2001).
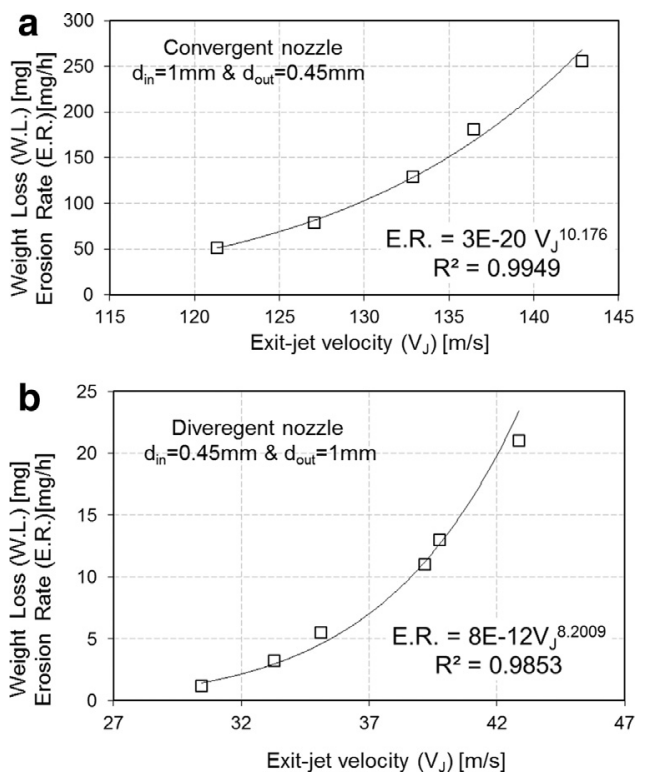

Fig. 11. Influence of the exit jet velocity on the erosion rate: (a) convergent nozzle and (b) divergent nozzle (working conditions are in Table 2).

In the case of divergent nozzle and low jet velocity the plastic deformation is localized in a ring shaped area. With time the deformation becomes more pronounced and, due to the firm contact between the specimen and the holder, the area of plastic deformation will spread (Fig. 10(b), $V_{\mathrm{J}}$ is $30.5 \mathrm{~m} / \mathrm{s}$ ). After a longer period, the cavitation cloud attacks an already roughened surface which leads to inhomogeneity in the stresses at the surface of the specimen. At some point, in areas characterized by sharp edges, further localization of the stresses occurs, leading to rupture, i.e., to the formation of the first pit (for example Fig. 10(b), $V_{\mathrm{J}}$ is $35.1 \mathrm{~m} / \mathrm{s}$ ). Further exposure to cavitation just broadens the initially formed pits, both in their width and depth (Fig. 10(b), $V_{\mathrm{J}}$ is $42.9 \mathrm{~m} / \mathrm{s}$ ). The collapses introduce very high pressure on the water trapped within the pits, resulting in stresses high enough to produce cracks and rupture inside the pits, which leads to the propagation of the damage. The first registered erosion, which is assumed to be analogous to the crack initiation step, will trigger the localization of deeper erosion in one ring. This process is more pronounced with decreasing cavitation number in the case of convergent nozzle as in Fig. 8(a).

The comparison between the convergent and the divergent nozzle leads to the following remarks:

(i) The erosion area in the case of the divergent nozzle was much smaller compared to the convergent one. In the case of the divergent nozzle, there could be found a very small area at the centre of the eroded specimen which had practically not been affected by the cavitation, while in the case of the convergent one, this area does not appear clearly or can be neglected. Additionally, in the case of the divergent nozzle, the erosion was shifted to the centre by increasing the jet velocity. (ii) The erosion process was strongly depending on the exit jet velocity in both cases. 
a
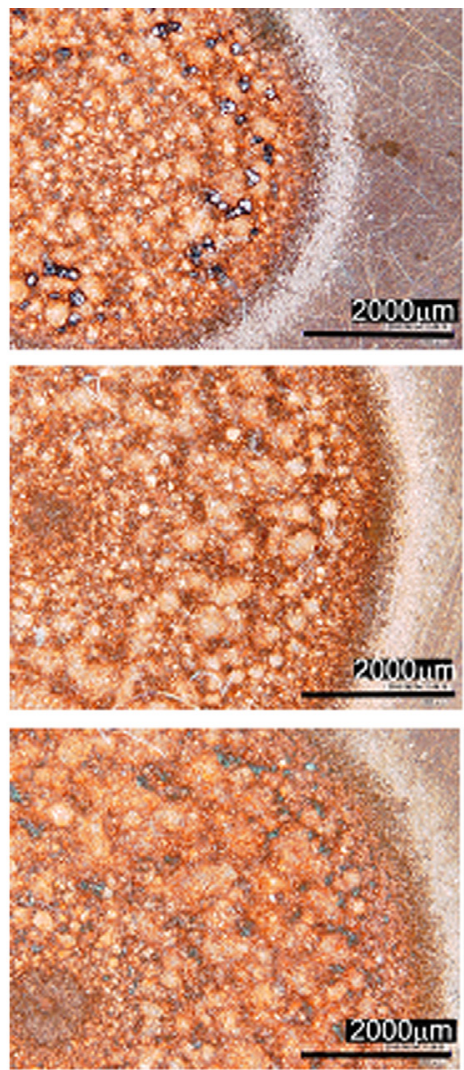

b
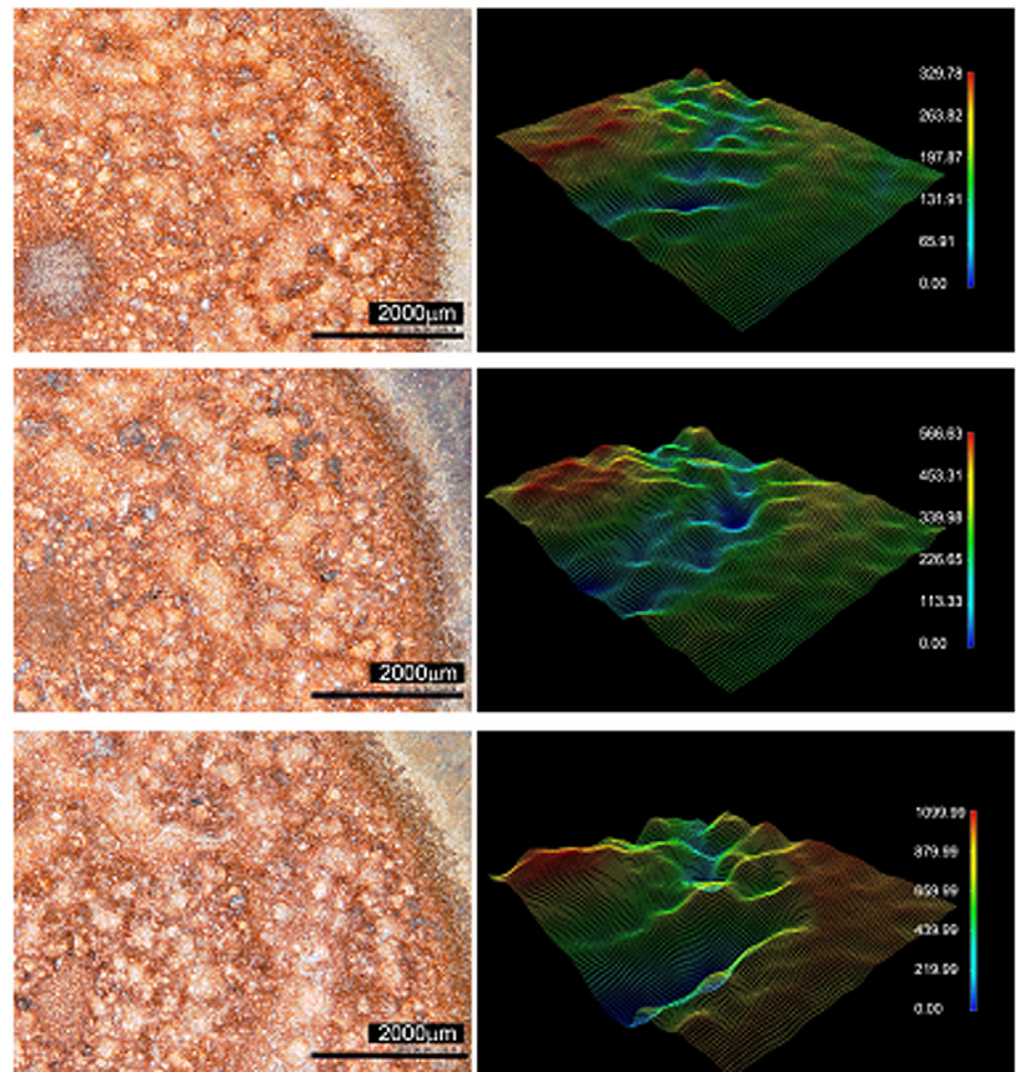

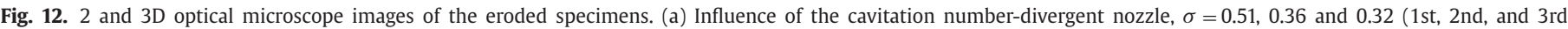
rows, respectively). (b) Influence of the exit jet velocity convergent nozzle: $V_{\mathrm{J}}=121.3,132.9$, and $142.8 \mathrm{~m} / \mathrm{s}$ ( $1 \mathrm{st}$, $2 \mathrm{nd}$, and $3 \mathrm{rd}$ rows, respectively) 
Table 3

Working conditions - the influence of the upstream pressure.

\begin{tabular}{lllllll}
\hline$P_{1}$ [bar] & $P_{2}$ [bar] & $Q\left[\mathrm{~m}^{3} / \mathrm{s}\right]$ & $V_{\mathrm{j}}[\mathrm{m} / \mathrm{s}]$ & $\sigma$ [dimensionless] & W.L. [mg] & E.R. [mg/h] \\
\hline 146.5 & 3.21 & $1.81 \mathrm{E}-05$ & 113.7991 & 0.049475 & 6.6 & 13.2 \\
160 & 3.23 & $1.89 \mathrm{E}-05$ & 119.0316 & 0.045503 & 10.4 & 20.8 \\
170 & 3.26 & $1.952 \mathrm{E}-05$ & 122.7582 & 0.04318 & 30 & 60 \\
186 & 3.28 & $2.043 \mathrm{E}-05$ & 128.5061 & 0.039645 & 86 & 172 \\
206 & 3.31 & $2.152 \mathrm{E}-05$ & 135.3465 & 0.036066 & 128 & 256 \\
216 & 3.33 & $2.204 \mathrm{E}-05$ & 138.6385 & 0.034581 & 157 & 314 \\
226 & 3.35 & $2.255 \mathrm{E}-05$ & 141.8542 & 0.033229 & 213 & 426 \\
235 & 3.36 & $2.301 \mathrm{E}-05$ & 144.6897 & 0.032035 & 246.9 & 493.8 \\
\hline
\end{tabular}
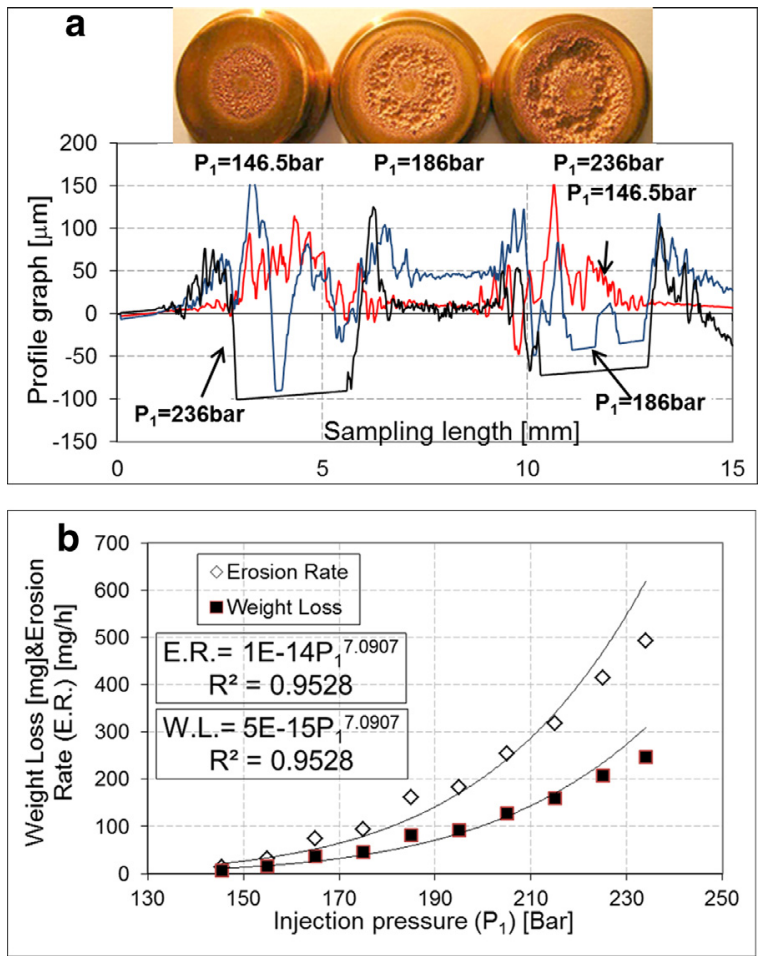

Fig. 13. Influences of the upstream pressure on (a) surface profile, and (b) erosion rate - convergent nozzle.

The differences that appeared between the two cases were only in the magnitude of the erosion and the degree of roughness. (iii) For the nozzle mounted in correct direction (divergent), the change in the range of exit-jet velocity was less pronounced than in the case of the nozzle mounted in the opposite in direction (convergent nozzle). The opposite direction was proposed by the authors to achieve a high cavitation intensity at low injection pressure (i.e. using low power) as compared with the correct direction, which was proposed by the manufacturer. These points can be also noted regarding the results of the influence of cavitation number on the erosion process (Fig. 8(a) and (b)).

In order to gain more insight regarding the effect of the nozzle geometry, exit jet velocity and cavitation number on the degree of the erosion, the attacked specimens were submitted to digital microscopy (Hirox-Digital Microscope KH-7700). Two and three dimensional images were created, which are presented in Fig. 12(a) and (b). The influence of tested parameters can be understood easily from the presented images. The investigation of the damaged samples for all test conditions has many rings with different degree of damage (roughness). These groups of rings are related to how the bubbles are distributed according to their strength and density in the jet cross-sections and to the pressure distribution around, near and on the target surface.
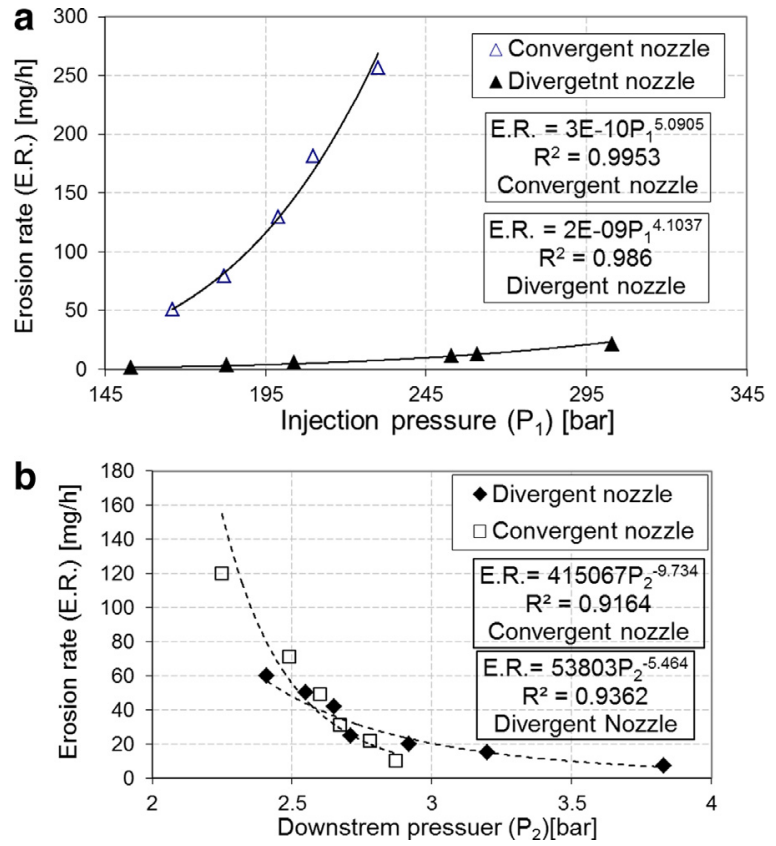

Fig. 14. (a) Influence of the downstream pressure on erosion process, exposure time $t=1 \mathrm{~h}$. Convergent nozzle $\left(V_{\mathrm{J}}=161 \mathrm{~m} / \mathrm{s}\right)$, divergent nozzle $\left(V_{\mathrm{J}}=30 \mathrm{~m} / \mathrm{s}\right)$ (see Table 1). (b) Influence of the upstream pressure $P_{1}$ on the erosion process, exposure time $t=1 \mathrm{~h}$. Convergent nozzle: $\sigma=0.0245$, divergent nozzle: $\sigma=1.04$ (see Table 2).

\section{Influence of the up and downstream pressures}

In order to investigate the influence of the upstream pressure on the jet behaviour and on the erosion process $\mathrm{Cu}$ specimens were exposed to the cavitating jet for $0.5 \mathrm{~h}$ at different upstream pressures. The value of $P_{1}$ was purposely varied, while the other parameters (cavitation number, downstream pressure $P_{2}$, exit jetvelocity) were left without any kind of control in order to determine how the upstream pressure affects these parameters. The distance between the specimens and the nozzle exit was fixed $\left(x / d_{\text {out }}=57.044\right)$, as was the water temperature. The nozzle was convergent. Table 3 shows the employed conditions. The investigation revealed that the erosion process was accelerated and the erosion area increased because the energy imparted on the attacked zone was increased as the injection (upstream) pressure increased (Soyama, 2011a; Choi et al., 2012; Hutli et al., 2010; Soyama et al., 2001, 2012; Minguan et al., 2013; Yamaguchi and Shimizu, 1987; Galeck et al., 2001). Surface profiles of the attacked specimens are presented in Fig. 13(a). The relations between the erosion rate/weight loss and upstream pressure are power relations as presented in Fig. 13(b). The observed results may be attributed to many reasons such as the velocity effect and the cavitation intensity effect. As the upstream pressure increases, both the cavitation intensity and the jet velocity increase (impact rate 
increases). Fig. 14 presents the influence of upstream pressure on the erosion rate, while the rest of parameters are kept constant (see Table 2- influence of exit jet velocity). The comparison between Figs. 13(b) and 14 reveals that, in both the relation between the erosion rate and upstream pressure is a power relation $\left(E . R .=A * P_{1}^{B}\right)$ where $A$ and $B$ are constants. These constants in Fig. 13(b) are not equal to those in Fig. 14(a) because of the reasons mentioned earlier. The interpretation of the curves behaviour in Fig. 14(a) is the same as that mentioned earlier for the influence of exit jet velocity. For downstream pressure Fig. 14(b) shows the relation between the erosion rate and downstream pressure.

In general, the results obtained in this work are in good agreement with previously published results, see for example, Choi et al. (2012), Soyama et al. (1994, 1995, 2009, 2012), Hutli et al. (2010), Soyama (2005), Kato and Shimomura (2001) and Nishimura et al. (2012). Although we have to note that in some of the cited cases the jet velocity was changed only via changing the nozzle diameter and geometry (the upstream and downstream pressures were not changed significantly, as it was reported by Kato and Shimomura, 2001).

\section{Conclusion}

Based on the results a better understanding regarding the behaviour of the cavitation jet and the influencing parameters can be established. In addition, simple mathematical relations between the erosion rate and tested parameters were found. In general, the followings points can be concluded:

- As the cavitation number decreases, the mass loss, the erosion rate, and the eroded area increase. The cavitation number has a strong influence on the cavitation intensity and on the distribution and strength of the bubbles in the jet coordinate directions $(r, x)$. In addition, it influences the penetration of the jet and the jet spreading angle.

- As the exit-jet velocity increases, the mass loss, erosion rate and eroded area increase. The exit-jet velocity has a large influence on the cavitation intensity and on the distribution and strength of the bubbles in the jet coordinate directions. In addition, it also influences the penetration of the jet, the jet spreading angle and the frequency of impact.

- The configuration of the nozzle (divergent or convergent) significantly influences the intensity of the cavitating jet produced by the cavitating jet generator, the jet width, the jet spreading angle and the distribution of the bubbles.

- The nozzle geometry dominates all the other parameters that may affect cavitation erosion because it governs the most important parameters ( $\sigma$ and $V_{\mathrm{J}}$ ).

- The distribution of the bubbles along the jet path and along its radial cross-section is a function of many parameters, such as up and downstream pressures ( $P_{1}$ and $P_{2}$, respectively), the nozzle diameter, the nozzle geometry and the flow pattern.

- The presence of various rings with differences in both width and in roughness on the surface of the attacked specimens is a good evidence for the previous points.

- The relationship between jet performance parameters and the erosion rate or weight loss can be described by relatively simple empirical mathematical formulas.

- The relation between the erosion rate and the tested parameters are power relations.

- In general, the results obtained in this work are in good agreement with previously published results.

\section{Acknowledgement}

The authors would like to express their thanks to the University of Kragujevac - Faculty of Mechanical Engineering, Serbia for assistance with the surface profile measurements. The first author is grateful for the Ministry of Science in Libya, for support through his research activity. Libyan government paid to EPFL-LMH for using the jet facility. Attila Bonyár is grateful for the support of Bolyai János Scholarship of the Hungarian Academy of Sciences.

\section{References}

Agrawal, A., Prasad, A., 2003. Measurements within vortex cores in a turbulent jet Trans. ASME J. Fluids Eng. 125, 561-568.

Bass, A., et al., 2008. Molecular dynamics of extreme mass segregation in a rapidly collapsing bubble. Phys. Rev. Lett. 101, 234-301.

Benajes, J., et al., 2004. Analysis of the influence of diesel nozzle geometry in the injection rate characteristic. Trans. ASME J. Fluid Eng. 126, 63-71.

Choi, J.K., et al., 2012. Scaling of cavitation erosion progression with cavitation intensity and cavitation source. Wear 278-279, 53-61.

Dynaflow, Inc., 2016, Research \& Development in Applied Sciences http://www. dynaflow-inc.com/Fluid_Gallery/Vortex-Ring-Gallery.htm

Emil, AB, et al., 2001. Dynamics of laser-induced cavitation bubbles near an elastic boundary. J. Fluid Mech. 433, 251-281.

Field, J.E., et al., 2012. Cavitation in impacted drops and jets and the effect on erosion damage thresholds. Wear 290-291, 154-160

Fujikawa, S., Takasugi, N., Peng, G., 1998. Cavitation characteristics of submerged water jet. In: Proceedings of the 3rd International Symposium on Cavitation Grenoble. France.

Galeck, G., et al., 2001. Limitations to the use of water jet in concrete substrate preparation. In: Proceedings of the WATA-American Water-Jet Conference, Paper 35. Minneapolis, Minnesota.

Hutli, E., Nedeljkovic, M., Radovic, N., 2008. Mechanics of submerged jet cavitating action: material properties, exposure time and temperature effects on erosion. Arch. Appl. Mech. 78, 329-341.

Hutli, E., Nedeljkovic, M., Vojislav, L., 2010. An experimental investigation of cavitating jet dynamic power and cavitation intensity. In: Proceedings of the ASME-International Mechanical Engineering Congress and Exposition on Fluid Flow, Heat Transfer and Thermal Systems, Vancouver, British Columbia, Canada ISBN: 978-0-7918-44441.

Hutli, E., Nedeljkovic, M., 2008. Frequency in shedding/discharging cavitation clouds determined by visualization of a submerged cavitating jet. Trans. ASME J. Fluids Eng. 130, 561-568.

Hutli, E., et al., 2013. Influences of hydrodynamic conditions, nozzle geometry on appearance of high submerged cavitating jets. Therm. Sci. 17, 1139-1149.

Karimi, A., Martin, J.L., 1986. Cavitation erosion of materials. Int. Met. Rev. 31, 1-26.

Kalumuck, K.M., et al. (1994): Evaluation of Cavitating Water Jets for Deep Hole Drilling in Hard Rock. Technical Report 93012-01. Dynaflow, Inc., March 1994.

Katepalli, R.S., 1999. Fluid turbulence. Rev. Mod. Phys. 71, 383-395.

Kato, H., Shimomura, Y.: Erosive intensity measurements of cavitating jet with various configurations. In: Proceedings of International Symposium on Cavitation, CAV2001, Session A4.002 (2001).

Minguan, Y., et al., 2013. Effect of geometrical parameters on submerged cavitation jet discharged from profiled central body nozzle. Chin. J. Mech. Eng. 26, 1-7. doi:10.3901/CJME.2013

Moss, W.C., et al., 1994. Hydrodynamic simulations of bubble collapse and picosecond sonoluminescence. Phys. Fluids 6, 2979-2985.

Nurick, W., 1976. Orifice cavitation and its effect on spray mixing. Trans. ASME J. Fluids Eng. 98, 681-687.

Nishimura, S., Takakuwa, O., Soyama, H., 2012. Similarity law on shedding frequency of cavitation cloud induced by a cavitating jet. J. Fluid Sci. Technol. 7, 405-420.

Okada, T., et al., 1995. Relation between impact load and the damage produced by cavitation bubble collapse. Wear 184, 231-239.

Sato, K., Yasuhiro, S., 2003. Unstable cavitation behavior in a circular cylindrical orifice flow. JSEM Int. J. Ser. B 45, 638-645.

Soyama, H., 2011. Enhancing the aggressive intensity of a cavitating jet by means of the nozzle outlet geometry. Trans. ASME J. Fluids Eng. 133, 1-11. doi:10.1115/1. 4004905.

Soyama, H., Nishizawa, K., Miami, M., 2009. Enhancement of cavitation aggressivity around a cavitating jet by injecting low-speed water jet for cavitation peening. In: Proceedings of the 7th International Symposium on Cavitation, CAV2009. Ann Arbor, Michigan, USA, pp. 1-10.

Soyama, H., et al., 2012. Optimum injection pressure of a cavitating jet for introducing compressive residual stress into stainless steel. J. Power Energy Syst. 6 , 63-75. doi:10.1299/jpes.6.63

Soyama, H., Asahara, M., 1999. Improvement of the corrosion resistance of a carbon steel surface by a cavitating jet. J. Mater. Sci. Lett. 18, 1953-1955.

Soyama, H., Lichtarowicz, A., Lambpard, D., 1998. Useful correlations for cavitating jets. In: Proceedings of the 3rd International Symposium on Cavitation, vol. 2. Grenoble, France, pp. 147-156.

Soyama, H., et al., 1998. A new calibration method for dynamically loaded transducers and its application to cavitation impact measurement. Trans. ASME J. Fluids Eng. 120, 712-718.

Soyama, H., 2011. Luminescent spots induced by a cavitating jet. In: Proceedings of ASME-JSME-KSME Joint Fluids Engineering Conference. Hamamatsu, Shizuoka, Japan, pp. $1-8$

Soyama, H., 2005. High-speed observation of a cavitating jet in air. Trans. ASME J. Fluids Eng. 127, 1095-1101. 
Soyama, H., et al., 1994. High-speed cavitation-cloud observations around high-speed submerged water jets. In: Proceedings of the 2nd International Symposium on Cavitation. Tokyo, Japan, pp. 225-230.

Soyama, H., et al., 1995. High-speed cavitation-cloud observations around high-speed submerged water jets. JSME Int. J. Ser. B. 38, 245-251.

Soyama, H., Kumano, H., Saka, M., 2001. A new parameter to predict cavitation erosion. In: Proceedings of International Symposium on Cavitation, CAV2001, Session A3.002 http://caltechconf.library.caltech.edu/109/ .

Sridhar, G., Katz, J., 1995. Drag and lift forces on microscopic bubbles entrained by a vortex. Phys. Fluids 7, 389-399.

Sun, Z., Kang, X.Q Wang, X.H., 2005. Experimental system of cavitation erosion with water-jet. Mater. Des. 26, 59-63.
Vijay, M., Zou, C., Tavoularis, S., 1991. A study of the characteristics of cavitating water jets by photography and erosion. In: Proceeding of the 10th International Conference on Jet Cutting Technology. Elsevier Science Publishers Ltd., pp. 37-67.

Wu, C., Roberts, P., 1993. Shock wave propagation in a sonoluminescing gas bubble. Phys. Rev. Lett. 70 (22), 3424-3427.

Yamaguchi, A., Shimizu, S., 1987. Erosion due to impingement of cavitating jet. Trans. ASME J. Fluids Eng. 109, 442-447.

Zhou, Y.K., Hammitt, F.G., 1983. Cavitation erosion incubation period. Wear 86, 299-313. 